\title{
Osteogenic ability using porous hydroxyapatite scaffold-based delivery of human placenta-derived mesenchymal stem cells
}

\author{
XIAOHUA REN $^{1 *}$, QINGWEI WANG $^{2 *}$, CHUNHUI LIU $^{1}$, QIAN ZHAO $^{3}$, JIAJUN ZHENG $^{4}$, \\ KUN TIAN $^{1}$, HUIJUAN XU ${ }^{2,5}$ and YANDONG MU ${ }^{1}$ \\ ${ }^{1}$ Stomatology Department, Sichuan Provincial People's Hospital, \\ University of Electronic Science and Technology of China, Chengdu, Sichuan $610072 ;{ }^{2}$ Institute of Chengdu Biology and \\ Sichuan Translational Medicine Hospital, Chinese Academy of Sciences, Chengdu, Sichuan 610041 ; $^{3}$ Shuangliu Hospital of \\ Traditional Chinese Medicine, Chengdu, Sichuan 610000; ${ }^{4}$ Southwest Medical University, Luzhou, Sichuan 646000; \\ ${ }^{5}$ The University of Chinese Academy of Sciences, Beijing 100039, P.R. China
}

Received February 15, 2020; Accepted June 11, 2021

DOI: $10.3892 / \mathrm{etm} .2021 .10525$

\begin{abstract}
Previous preliminary studies have suggested that hydroxyapatite with a grooved structure (HAG) scaffold has good osteogenic potential. This type of scaffold may aid osteogenesis during the repair of large maxillofacial bony defects. The ectopic osteogenic effect and underlying mechanism were further studied using porous HAG scaffold-based delivery of human placenta-derived mesenchymal stem cells (hPMSCs). A total of 18 dogs were randomly allocated into a HAG scaffold group and a HAG scaffold-based hPMSC (HAG/hPMSC) group, and three scaffolds were implanted into the dorsal muscle of each dog. Samples were taken for subsequent analysis and tested 4, 8 and 12 weeks following heterotopic implantation. H\&E staining was used to study the osteogenic effect in dog dorsal muscles, and RNA sequencing (RNA-seq) was used for exploring the underlying osteogenic mechanism. The osteogenic ability and effector of the HAG/hPMSC group were significantly greater than those of the HAG scaffold group at 4 weeks after implantation. After 12 weeks, a mature bone plate structure was seen in the HAG/hPMSC group. RNA-seq demonstrated that various osteogenesis-related pathways participated at different stages of metabolism, and that the expression of collagen-1 and runt-related transcription factor 2 increased with implantation time. The present study preliminarily focused on the ectopic osteogenic effect of the
\end{abstract}

Correspondence to: Professor Yandong $\mathrm{Mu}$, Stomatology Department, Sichuan Provincial People's Hospital, University of Electronic Science and Technology of China, 32 West Section 4, First Ring Road, Chengdu, Sichuan 610072, P.R. China

E-mail: muyd@uestc.edu.cn

*Contributed equally

Key words: hydroxyapatite scaffold, human placenta-derived mesenchymal stem cell, osteogenesis, delivery porous HAG scaffold-based delivery of hPMSCs in vivo, which may be helpful for the improved application of HAG scaffolds in the future.

\section{Introduction}

Maxillary defect or deficiency are the most common problems in dental implantation, especially in the treatment of patients with extensive jawbone defects caused by trauma or tumor resection (1). Due to the rapid development of material science, bone tissue engineering has attracted wide attention, and extensive research has been carried out on the subject. Bone tissue engineering strikes a balance between the advantages and disadvantages of autogenous bone and allogeneic bone (2). Artificial bone substitute materials are based on bone tissue engineering using scaffolds, growth factors and stem cells; these have good histocompatibility and reproducibility, and are expected to be good substitutes for bone defect repair (3).

Hydroxyapatite (HA), as an inorganic mineral, is the main component of natural bone inorganic salts, and an indispensable inorganic component of the human skeleton (4). Research demonstrates that $50 \%$ of the human skeleton is comprised of homogeneous inorganic HA (4). The physicochemical properties, chemical composition and crystal structure of HA are very similar to human bone and are highly safe for humans. The results of our previous study demonstrated that porous HA with a grooved structure (HAG) scaffolds enhanced osteogenesis in vivo and in vitro (5).

Transcriptome sequencing is a technique for determining the expression level of the transcriptome, and is widely used in research (6). RNA sequencing (RNA-seq) is a high-throughput sequencing assay that can provide detailed information regarding the working mechanisms underlying the target tissue or the molecular pathobiology of a disease (7-11). Thus, RNA-seq plays an important role in studying the mechanisms underlying osteogenesis. High-throughput transcriptome profiling was used to further understand the molecular mechanism underlying lithium in regulating the osteogenic fate of human mesenchymal stem cells (hMSCs), when stimulated with lithium for 7 days (12). 
According to the International Society for Cellular Therapy (ISCT) (13), mesenchymal stem cells (MSCs) must satisfy three requirements: i) MSCs must be plastic-adherent when maintained in standard culture conditions; ii) MSCs must express CD105, CD73 and CD90, and lack the expression of CD45, CD34, CD14 and human leukocyte antigen (HLA)-DR surface molecules and either CD14 or CD11b, and either CD79a or CD19; and iii) MSCs must differentiate to osteoblasts, adipocytes and chondroblasts in vitro (13). The criteria do not support the purification of homogenous MSC populations. The isolation of MSCs according to ISCT criteria produces heterogeneous, non-clonal cultures of stromal cells containing stem cells with different multipotent properties, committed progenitors and differentiated cells. Currently, MSCs can be isolated from multiple tissues, such as placenta $(14,15)$.

In the present study, the RNA-seq technique was used to study the osteogenic mechanism underlying porous HA scaffold-based delivery of human placenta-derived (hP) MSCs in the dorsal muscles of dogs. Samples were taken for sequencing 4, 8 and 12 weeks after heterotopic implantation. The association between the expression and time of different genes was identified by analyzing differentially expressed genes, and the osteogenesis mechanism underlying the porous HAG scaffold-based delivery of hMSCs was investigated using results of transcriptome sequencing. The novelty of the present study was demonstrated by the analysis of the biological properties of the porous HAG scaffold-based delivery of hMSCs via transcriptomics, which provided novel applications of this cell type in osteogenesis research.

\section{Materials and methods}

Preparation of cell-adhered HAG scaffolds for transplantation. Cell culture conditions were the same as in our previous study (5): High glucose DMEM (Thermo Fisher Scientific, Inc.) containing 10\% FBS (Thermo Fisher Scientific, Inc.), $20 \mathrm{mmol} / \mathrm{l} \beta$-glycerophosphate, $50 \mathrm{~g} / \mathrm{ml}$ vitamin $\mathrm{C}$ and $10 \mathrm{~mol} / \mathrm{l}$ dexamethasone at $37^{\circ} \mathrm{C}$ in $5 \% \mathrm{CO}_{2}$. The immortalized hPMSCs used in this study originated from the State Key Laboratory of Biological Therapy at Sichuan University (provided by the National Experimental Cell Resource Sharing Platform, http://www.cellresource.cn/). The fifth generation of hPMSCs in the exponential growth period were counted. According to the counting results, high glucose DMEM was added until the cell density reached $2 \times 10^{5} / \mathrm{ml}$. According to our previous study (5), cells were analyzed using a flow cytometer (FACSAria III; BD Biosciences) with related antibodies (all dilutions, 1:100; all from Abcam) for the following surface markers: HLA-DR (cat. no. ab92511), CD11b (cat. no. ab133357), CD14 (cat. no. ab183322), CD19 (cat. no. ab134114), CD34 (cat. no. ab81289), CD44 (cat. no. ab189524), CD45 (cat. no. ab40763), CD73 (cat. no. ab202122), CD79 (cat. no. ab134147), CD90 (cat. no. ab23894) and CD105 (cat. no. ab231774) (16,17). A total of $5 \times 10^{5}$ cells were cultured in a flow tube at $37^{\circ} \mathrm{C}$, after which cells were re-suspended into monocytes using $1001 \mathrm{X}$ PBS and antibodies $(1 \mu \mathrm{l})$ were added. Samples were incubated at $4^{\circ} \mathrm{C}$ for $30 \mathrm{~min}$ in the dark and analyzed using FlowJo 10.6.2 software (BD Biosciences).
The cells were directly dripped onto the surface of the porous HAG scaffold until the material was just infiltrated by the cell suspension. Following incubation for $3 \mathrm{~h}$, the HAG scaffold was turned over, cell suspension droplets were added to the other side of the scaffold and culture medium (high glucose DMEM) was added following a further $3 \mathrm{~h}$ in the incubator. Tissue engineered bone was cultured in a constant temperature incubator at $37^{\circ} \mathrm{C}$ on a six-well plate for 7 days, and the solution was changed once every 1-2 days. hPMSCs were cultured in low-glucose DMEM supplemented with 10\% FBS and 1\% penicillin-streptomycin in a $5 \% \mathrm{CO}_{2}$ atmosphere at $37^{\circ} \mathrm{C}$.

Preparation and characterization of the scaffolds. The HAG scaffolds were used in this study as described by our previous work (5).

Experimental animals and implantation. All animal experiments were conducted according to the protocols approved by the Animal Care and Use Committee of Sichuan Provincial People's Hospital [Sichuan, P.R. China; approval no. 2019NSF(98)]. A total of 18 male beagles (age, 12 months old; weight range, $10-12 \mathrm{~kg}$ ) were selected as the experimental samples. Beagles were housed at $16-28^{\circ} \mathrm{C}$ (softened animal feed with milk and water were obtained freely from 8 a.m. to 5 p.m.) and under relative humidity of 40-80\%. They were exposed to a $12 \mathrm{~h}$ light/dark cycle and ammonia concentrations of $<14 \mathrm{mg} / \mathrm{m}^{3}$, which was administered to avoid the occurrence of disease. Beagles were provided by the Experimental Animal Center of Sichuan Provincial People's Hospital (Sichuan, P.R. China). All animal surgery was approved by the Institutional Animal Care and Use Committee and implemented according to relevant requirements. The beagles were used for a duration of 4 months in the present study. Pentobarbital sodium was injected intravenously into the forelimb for general anesthesia at a concentration of $30 \mathrm{mg} / \mathrm{kg}$. The animals were euthanized $1 \mathrm{month}$ after the experiment, using pentobarbital sodium injected intravenously into the forelimb at a concentration of $100 \mathrm{mg} / \mathrm{kg}$. The death of all animals was confirmed by cardiac arrest, respiratory arrest and loss of reflex. A total of 18 beagles were randomly allocated into one of the following groups, with 3 beagles/group: i) HAG group with stem cell implantation (HAG/hPMSC group) and ii) HAG group without stem cell implantation. The scaffolds were implanted separately into the dorsal muscle of each dog as described by the heterotopic implantation discussion in our previous study (5). The scaffold attached by muscle from each beagle was obtained, and RNA and protein were extracted for analysis by crushing with a grinder. A total of three scaffolds from the dorsal muscle of each dog were pooled together for RNA-seq. Western blotting analysis and qPCR were repeated three times.

$H \& E$ staining and western blot analysis. At 4, 8 and 12 weeks after surgery, the surface of the scaffold was washed with normal saline and placed into $10 \%$ neutral formaldehyde fixing solution at $4{ }^{\circ} \mathrm{C}$ overnight. New bone was identified using optical microscopy of H\&E-stained implanted materials. $\mathrm{H} \& \mathrm{E}$ staining can detect the formation of bone matrix, which can indicate osteogenic effects $(18,19)$. Images were captured using an optical microscope (CX23; Carl Zeiss AG). 
Following SDS-PAGE and membrane transfer, the membrane was incubated overnight at $4^{\circ} \mathrm{C}$ with $\operatorname{IgG}$ (Abcam; 1:300). Following incubation with the secondary antibody, buffer from the membrane was discarded and developing reagent was added. The PVDF membrane was rocked until development of bands was apparent. The membrane was washed three times using distilled water for $30 \mathrm{~min}$. The membrane was visualized using ChemiDocMP (Bio-Rad Laboratories, Inc.).

Isolation and purification of RNA. For mRNA deep sequencing, total RNA was extracted from the control and treated frozen samples using TRIzol ${ }^{\circledR}$ (Invitrogen; Thermo Fisher Scientific, Inc.) according to the manufacturer's instructions. The quality of total RNA was checked using an Agilent 2100 Bioanalyzer (Agilent Technologies, Inc.). Equal amounts of RNA samples from three control individuals were pooled to generate a mixed sample for the control subgroup, and the same was repeated for the treated subgroup.

cDNA synthesis and library construction for $m R N A$ deep sequencing. For mRNA sequencing, a cDNA library was constructed with a NEBNext ${ }^{\circledR}$ Ultra $^{\mathrm{TM}}$ RNA Library Prep kit (Illumina, Inc.). The concentration of the library was $>2$ $\mathrm{nM}$, indicating that the library was effective. Samples were diluted to $1 \mathrm{ng} / \mu \mathrm{l}$ and detected using a DNA Quantification kit (llumina, Inc.). Briefly, mRNA was purified from total RNA using poly-T oligo-attached magnetic beads. Fragmentation was carried out using divalent cations under elevated temperature in NEBNext First Strand Synthesis Reaction Buffer (5X) (Illumina, Inc.). Index codes were added to attribute sequences to each sample and 150 bp paired-end reads were generated. The obtained library was used for sequencing with Illumina Hiseq $^{\mathrm{TM}} 2500$ (Illumina, Inc.).

Reference genome and gene model annotation files were downloaded from genome websites (NCBI, https://www.ncbi. nlm.nih.gov/) directly. An index of the reference genome was built using Bowtie v2.2.3 (http://bowtie-bio.sourceforge. net/bowtie2/index.shtml), and paired-end clean reads were aligned to the reference genome using TopHat v2.0.12 (http://ccb. jhu.edu/software/tophat/manual.shtml). TopHat was selected as the mapping tool as it can generate a database of splice junctions based on the gene model annotation files. We used KOBAS software (http://kobas.cbi.pku.edu.cn/) to test the statistical enrichment of differential expression genes in KEGG pathway.

Reverse transcription-quantitative PCR (RT-qPCR). To validate the RNA-seq data, differentially expressed genes were selected for RT-qPCR analysis. All primers were designed by the Premier5.0 software (Premier Biosoft International; Table I). RNA was extracted using the TRIzol kit (Thermo Fisher Scientific, Inc.). cDNA was synthesized at $37^{\circ} \mathrm{C}$ for $15 \mathrm{~min}$ and $85^{\circ} \mathrm{C}$ for $5 \mathrm{sec}$ using the Prime Script ${ }^{\circledR}$ RT reagent kit with the gDNA Eraser kit (all from Takara Biotechnology Co., Ltd.). RT-qPCR was carried out with SsoFast $^{\mathrm{TM}}$ EvaGreen ${ }^{\circledR}$ Supermix (Bio-Rad Laboratories, Inc.). Two mRNAs were selected at random from upregulated, downregulated and non-altered genes for qPCR analysis using $\beta$-actin as a reference gene. The fold change from the qPCR was determined using the $2^{-\Delta \Delta C q}$ method (20). The RNAs of samples were reverse transcribed into cDNA in a
Table I. Primers for reverse transcription-quantitative PCR.

\begin{tabular}{llc}
\hline Name & \multicolumn{1}{c}{ Sequence (5'-3') } & Fragment, bp \\
\hline COL-1-F & AGGGGTCTCCATGGTGAGTT & 119 \\
COL-1-R & GAAGGACCTCGGCTTCCAAT & \\
Runx2-F & TTCCAGAATGCTTCCGCCAT & 110 \\
Runx2-R & AACTGCTGTGGCTTCCATCA & \\
$\beta$-actin-F & CAATACAACTCTCCACAACC & 281 \\
$\beta$-actin-R & CAGATAGCACCTTCAGCAC & \\
\hline
\end{tabular}

Col-1, collagen-1; Runx2, runt-related transcription factor 2; F, forward; $\mathrm{R}$, reverse.

Table II. Statistical anlaysis of the ratio of total area occupied by the newborn.

\begin{tabular}{lcc}
\hline Sample & Mean, $\%$ & Standard deviation, \% \\
\hline HAG-4w & 3.7 & 0.3 \\
HAG/hPMSC-4w & 5.2 & 0.5 \\
HAG-8w & 6.5 & 1.2 \\
HAG/hPMSC-8w & 5.1 & 0.3 \\
HAG-12w & 11.2 & 0.3 \\
HAG/hPMSC-12w & 12.3 & 0.4 \\
\hline
\end{tabular}

HAG, hydroxyapatite with a grooved structure; hPMSC, human placenta-derived mesenchymal stem cells; HAG/hPMSCs, HAG scaffold-based delivery of hPMSCs; w, weeks.

$20 \mu \mathrm{l}$ reaction containing $13 \mu \mathrm{l}$ RNA template, $2 \mu \mathrm{l}$ Oligo dT primer or microRNA sequence-specific primers $(10 \mu \mathrm{M}), 4 \mu \mathrm{l}$ of 5X PrimeScript Buffer and $1 \mu$ l PrimeScript RT Enzyme Mix I (Takara Bio, Inc.). For subsequent qPCR reactions using gene-specific primers, $1 \mu \mathrm{l}$ cDNA sample was amplified under the following cycle conditions: $94^{\circ} \mathrm{C}$ for $30 \mathrm{sec}$, followed by 39 cycles of $55^{\circ} \mathrm{C}$ for $20 \mathrm{sec}$ and $72^{\circ} \mathrm{C}$ for $20 \mathrm{sec}$.

Statistical analysis. All the statistical data were analyzed using Bonferroni ANOVA using SPSS 20.0 statistics software (IBM Corp.). All data are presented as the mean \pm SD. $\mathrm{P}<0.05$ was considered to indicate a statistically significant difference. All analyses were repeated three times. Gene expression used double-screening by q-value (adjusted P-value, $\leq 0.05$ ) and normalized $\log 2$ ratio ( $\mid \log 2$ ratio $l \geq 1$; Tables SI-SIII) according previous sequencing methods (21).

\section{Results}

Osteogenesis effects of the HAG-based delivery of hPMSCs. The beagles in the HAG/hPMSC experimental group had HAG scaffolds implanted with hPMSCs implanted in their dorsal muscles, while the HAG control group was implanted with the HAG scaffolds alone. At a total of 4, 8 and 12 weeks after the operation, two groups of scaffolds were obtained from the dorsal muscles of the beagles and labelled as follows: 


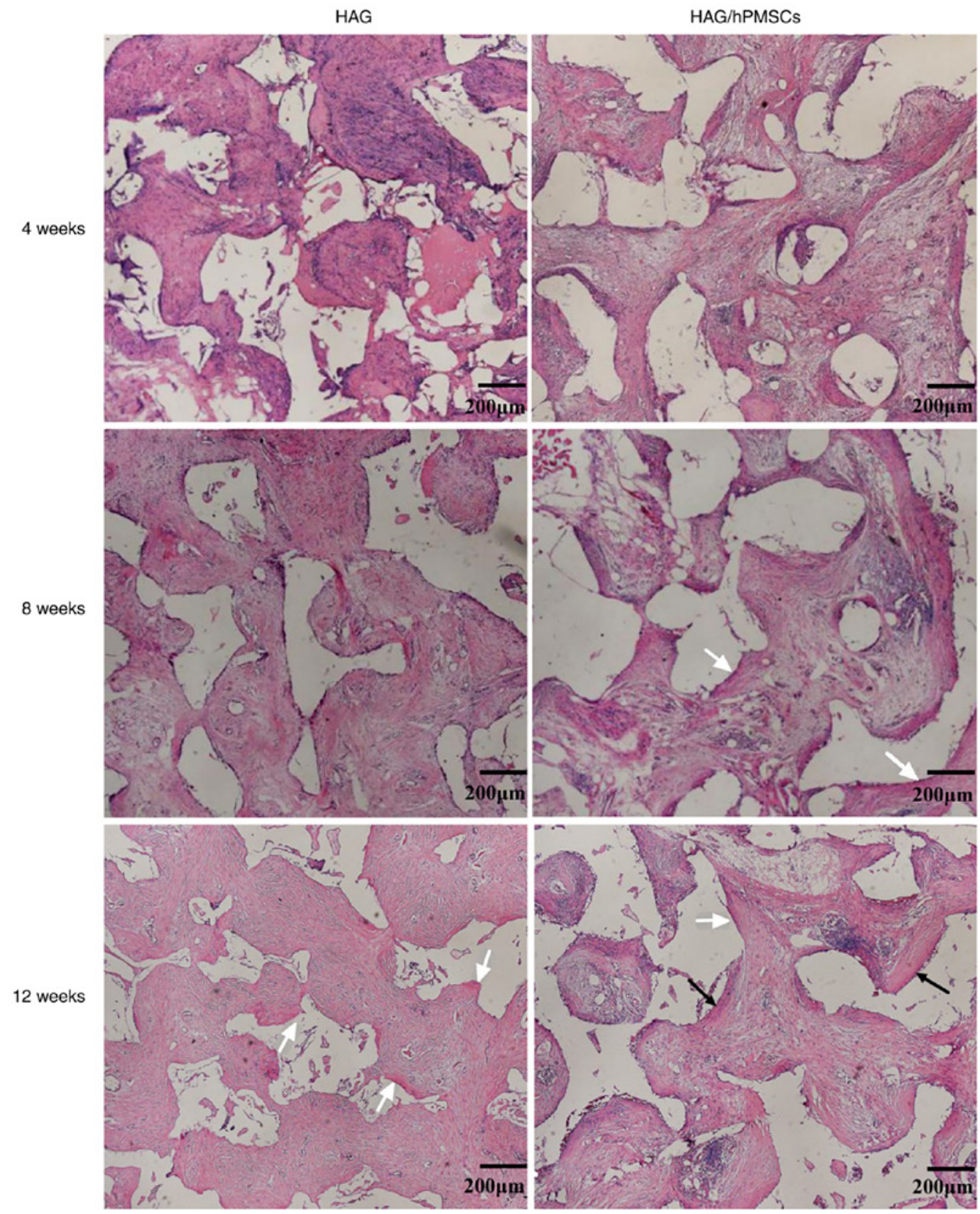

Figure 1. Bone formation of the scaffold analyzed using H\&E staining. Magnification, x100. White arrow, bone matrix; black arrow, mature bone plate structure. HAG, hydroxyapatite with a grooved structure; hPMSC, human placenta-derived mesenchymal stem cells; HAG/hPMSCs, HAG scaffold-based delivery of hPMSCs; w, weeks.

HAG-4 weeks (w), HAG-8w, HAG-12w and HAG/hPMSC-4w, HAG/hPMSC-8w and HAG/hPMSC-12w.

$\mathrm{H} \& \mathrm{E}$ staining demonstrated that a large number of fibroblasts grew and fibrous tissue formed in both groups at 4 weeks after implantation, while osteoid formation was found on the surface of scaffolds in the HAG/hPMSC-4w compared with the HAG-4w group (Fig. 1). After 8 weeks, the area of bone matrix in the $\mathrm{HAG} / \mathrm{hPMSC}-8 \mathrm{w}$ group was larger than that in the HAG group (Fig. 1). After 12 weeks, a large amount of bone matrix was formed in both groups, but mature bone plate structure was observed in the HAG/hPMSC-12w group (Fig. 1). The statistical results indicated that the osteogenic ability and effects of the HAG/hPMSC group were markedly greater than those of the HAG group (Table II).
Illumina sequencing and mapping to reference the genome. cDNA libraries were constructed for different RNA samples at different times of implantation, with one treatment and one control for Illumina sequencing. From these libraries, 49,398,934,53,012,698,51,462,894,60,674,628,53,302,412 and 48,165,136 raw reads were obtained from HAG/hPMSC-4w, HAG/hPMSC-8w, HAG/hPMSC-12w, HAG-4w, HAG-8w and HAG-12w, respectively, which generated 7.05, 7.59, 7.36, 8.65, 7.61 and $6.89 \mathrm{~Gb}$ of cleaned data. The schematic of Illumina deep sequencing and analysis is demonstrated in Table III.

High-quality reads were mapped to the coding sequences from the reference genome by Bowtie software, with the default parameters for the HAG group set as 48,759,095 $(84.59 \%), 43,082,474(84.9 \%)$ and $38,758,173(84.42 \%)$. For the HAG/hPMSC group, default parameters were set 


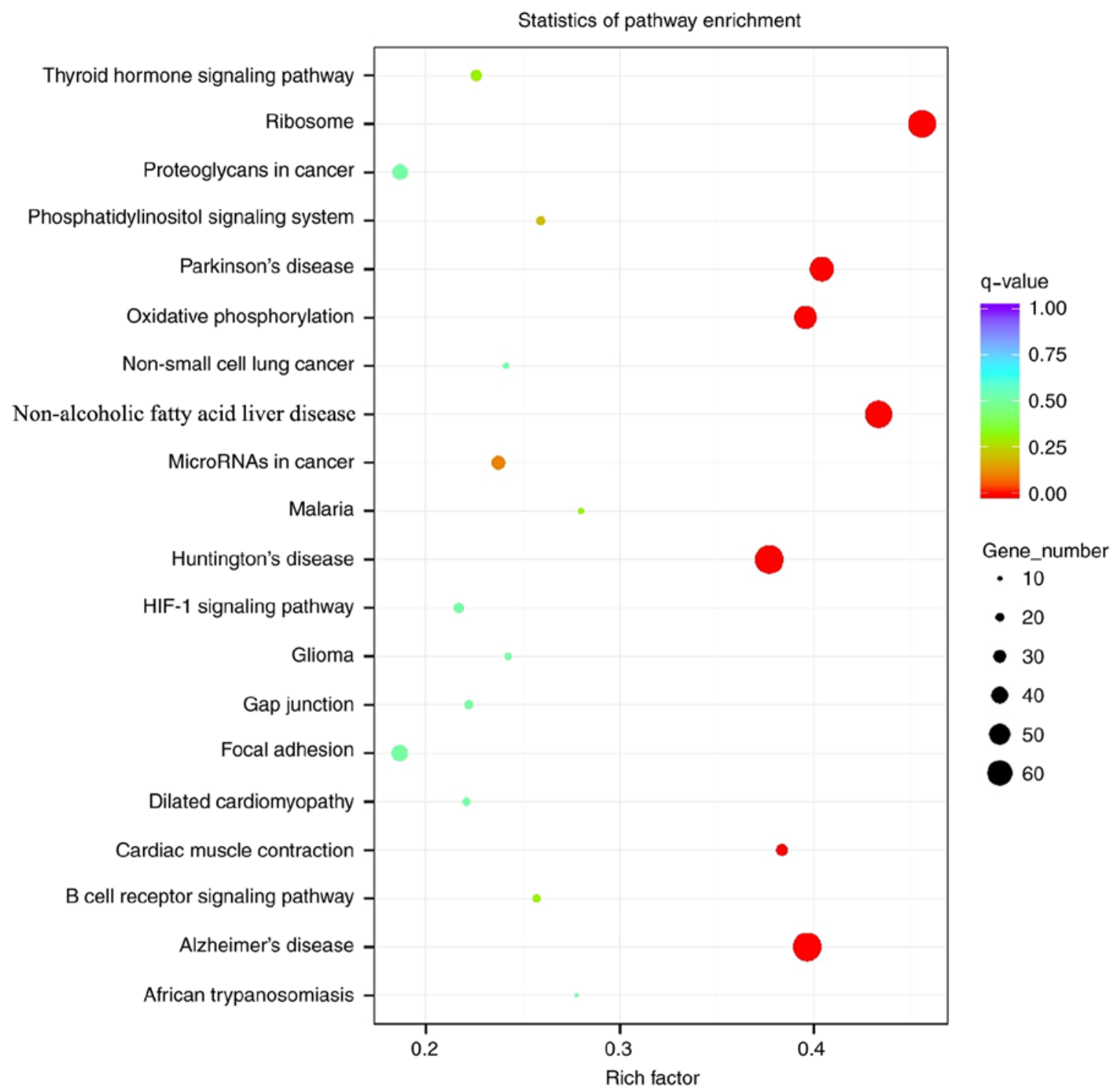

Figure 2. Kyoto Encyclopedia of Genes and Genomes enrichment pathways involved in the different stages of osteogenesis. The size of the solid circle represents the number of genes and the depth of the color represents the size of the q-value. HIF-1, hypoxia-inducible factor-1.

as $38,443,778(81.81 \%), 42,956,903(84.9 \%)$ and $41,701,352$ (84.94\%; Table IV).

Functions of differentially expressed genes. The sequencing data revealed that 1,539 (HAG-4w vs. HAG/hPMSC-4w), 1,187 (HAG-8w vs. HAG/hPMSC-8w) and 30 (HAG-12w vs. HAG/hPMSC-12w) genes that were expressed differently between the control and treated groups were related to osteogenesis responses. This was due to HAG scaffold-based delivery of hPMSCs under a double-screening using q-value (adjusted P-value, $\leq 0.05$ ) and normalized $\log 2$ ratio (llog2ratiol $\geq 1$; Tables SI-SIII) according previous sequencing methods (22).

Analysis of Kyoto Encyclopedia of Genes and Genomes (KEGG) enrichment demonstrated that the signal pathways enriched with differentially expressed genes differed during the all periods after implantation (Fig. 2). Fig. 2 summarizes all of the KEGG pathways, rather than the differences at each particular stage. At 4 weeks following implantation, the involved signaling pathways included 'extracellular matrix
(ECM)-receptor interaction' and 'PI3K-AKT signaling', compared with 'hematopoietic cell lineage', 'focal adhesion', 'cell adhesion molecules' (CAMs), 'protein digestion and absorption', 'platelet activation', 'B cell receptor signaling pathway' and 'osteoclast differentiation', which were involved at 8 weeks following implantation. At 12 weeks following implantation, the signaling pathways involved mainly included 'protein digestion and absorption', 'ECM-receptor interaction', 'AMP-activated protein kinase (AMPK) signaling' and 'osteoclast differentiation'.

Detection and verification of osteogenic genes. To validate the results of the Illumina sequencing, the osteogenic genes COL-1 and RUNX2 were selected for RT-qPCR analysis. The results revealed that $C O L-1$ (Fig. 3A) and RUNX2 (Fig. 3B) genes were expressed in HAG and HAG/hPMSC groups during heterotopic osteogenesis and that the expression increased with time. The gene expression in the HAG/hPMSC group was markedly upregulated compared with in the HAG group at all time points (Fig. 3). The results of western blot 
Table III. Statistics of mRNA sequencing reads.

\begin{tabular}{lccccccc}
\hline Sample & Raw reads & Clean reads & Clean bases, G & Error rate, \% & Q20, \% & Q30,\% & GC content, \% \\
\hline HAG-4w & $60,674,628$ & $57,642,840$ & 8.65 & 0.02 & 96.65 & 91.59 \\
HAG/hPMSC-4w & $49,398,934$ & $46,993,014$ & 7.05 & 0.02 & 96.88 & 92.03 & 50.29 \\
HAG-8w & $53,302,412$ & $50,746,542$ & 7.61 & 0.02 & 96.96 & 92.26 & 51.79 \\
HAG/hPMSC-8w & $53,012,698$ & $50,596,524$ & 7.59 & 0.02 & 96.83 & 91.94 & 50.55 \\
HAG-12w & $48,165,136$ & $45,911,572$ & 6.89 & 0.02 & 96.55 & 91.37 & 51.01 \\
HAG/hPMSC-12w & $51,462,894$ & $49,097,376$ & 7.36 & 0.02 & 96.81 & 91.88 & 50.58 \\
\hline
\end{tabular}

HAG, hydroxyapatite with a grooved structure; hPMSC, human placenta-derived mesenchymal stem cells; HAG/hPMSCs, HAG scaffold-based delivery of hPMSCs; w, weeks; Q20, the error probability for the identified bases during sequencing is 1\%; Q30, the error probability for the identified bases during sequencing is $0.1 \%$.
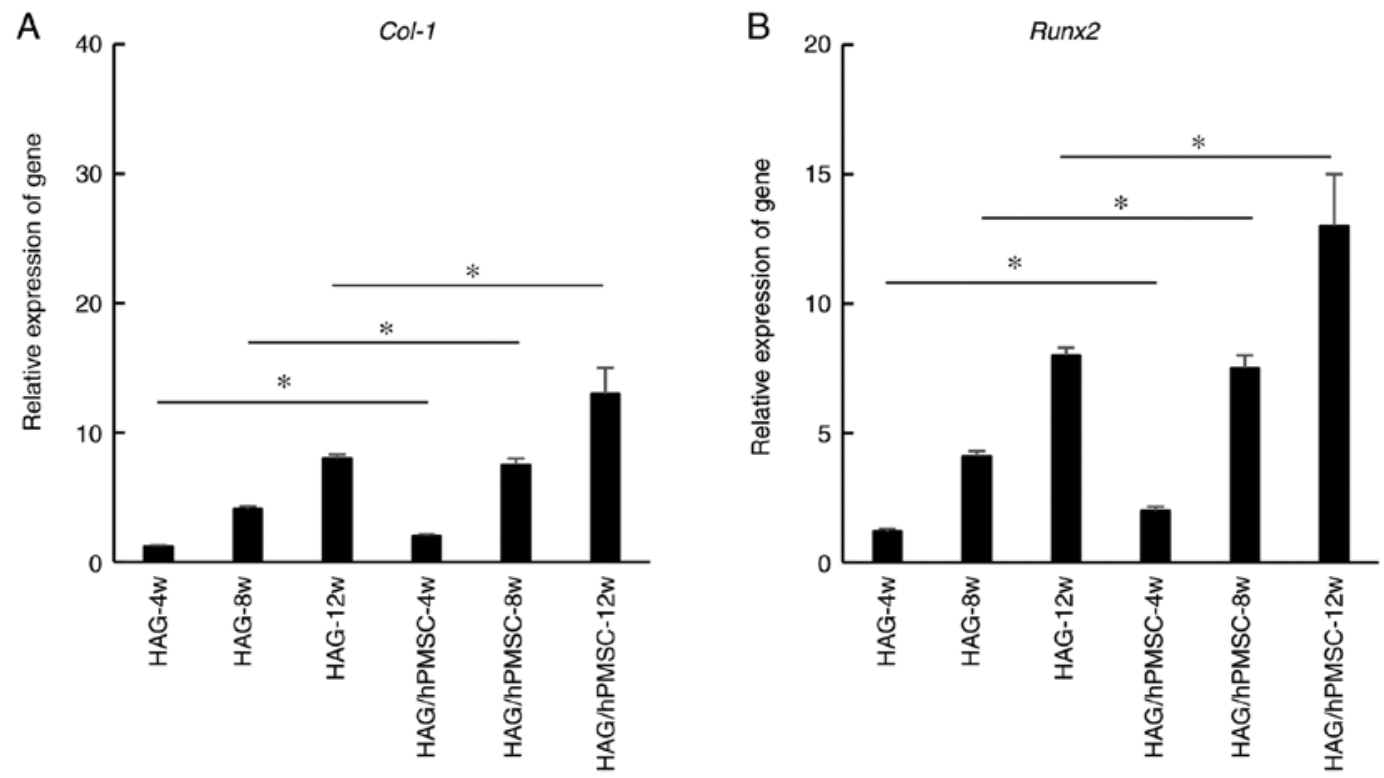

Figure 3. Relative expression of osteogenesis-associated genes. (A) Relative gene expression of $\mathrm{Col}-1$ at 4,8 and 12 weeks after implantation of two different scaffolds. (B) Relative gene expression of Runx 2 at different times after implantation of two different scaffolds. "P<0.05. Col-1, collagen-1; Runx2, runt-related transcription factor 2; HAG, hydroxyapatite with a grooved structure; hPMSC, human placenta-derived mesenchymal stem cells; HAG/hPMSCs, HAG scaffold-based delivery of hPMSCs; w, weeks.

analysis revealed that the expression levels of proteins COL-1 (Fig. 4A and B) and RUNX2 (Fig. 4A and C) were similar to those of the respective genes, but that the difference was not marked between each of the experimental groups.

\section{Discussion}

Tissue-engineered bone consists of seed cells, scaffolds and growth factors. Of the seed cells, bone marrow MSCs, embryonic stem cells and adipose stem cells are the most widely studied. Biomaterials with co-cultured stem cells exhibit good biocompatibility for increasing osteogenesis and thus offer potential as bone graft substitutes $(23,24)$. The seed cells used in the present study are MSCs derived from human placenta, which is an ideal source of seed cells for humans (25). To provide the growth factors required for tissue engineering, seed cells in the present study were co-cultured with porous scaffolds to explore whether this method effectively improved osteogenesis. The experimental results revealed that the scaffolds in the HAG/hPMSC group demonstrated improved heterotopic osteogenesis in beagle dorsal muscles. However, in contrast to other engineered cells, the stem cells used in the present study are derived from humans, allowing for more valuable application in future clinical use. The human-derived stem cells used in the present study provide the necessary growth factors for osteogenesis.

At 4 weeks following implantation, bone-like structure was also observed on the surface of the scaffold. Compared with canine stem cell-seeded collagen-hydroxyapatite scaffolds, the HAG/hPMSC scaffold used in the present study exhibited an advantage in promoting osteogenesis at 4 weeks compared with 3 months (26). Analysis of KEGG enrichment revealed that ECM-receptor interactions (27) were involved in the process of osteogenesis. Furthermore, the PI3K-AKT pathway, which inhibits apoptosis and increases cell survival rate (28), may have improved the surface compatibility between the scaffold and the muscle cells. At 8 and 12 weeks 
Table IV. Results of reads compared with the reference genome.

\begin{tabular}{lcrr}
\hline Sample & Total reads & Total mapped (\%) & Multiple mapped (\%) \\
\hline HAG-4w & $57,642,840$ & $48,759,095(84.59)$ & $1,043,484(1.81)$ \\
HAG/hPMSC-4w & $46,993,014$ & $38,443,778(81.81)$ & $885,270(1.88)$ \\
HAG-8w & $50,746,542$ & $43,082,474(84.9)$ & $866,880(1.71)$ \\
HAG/hPMSC-8w & $50,596,524$ & $42,956,903(84.9)$ & $844,023(1.67)$ \\
HAG-12w & $45,911,572$ & $38,758,173(84.42)$ & $701,480(1.53)$ \\
HAG/hPMSC-12w & $49,097,376$ & $41,701,352(84.94)$ & $742,653(1.51)$ \\
\hline
\end{tabular}

HAG, hydroxyapatite with a grooved structure; hPMSC, human placenta-derived mesenchymal stem cells; HAG/hPMSCs, HAG scaffold-based delivery of hPMSCs; w, weeks.

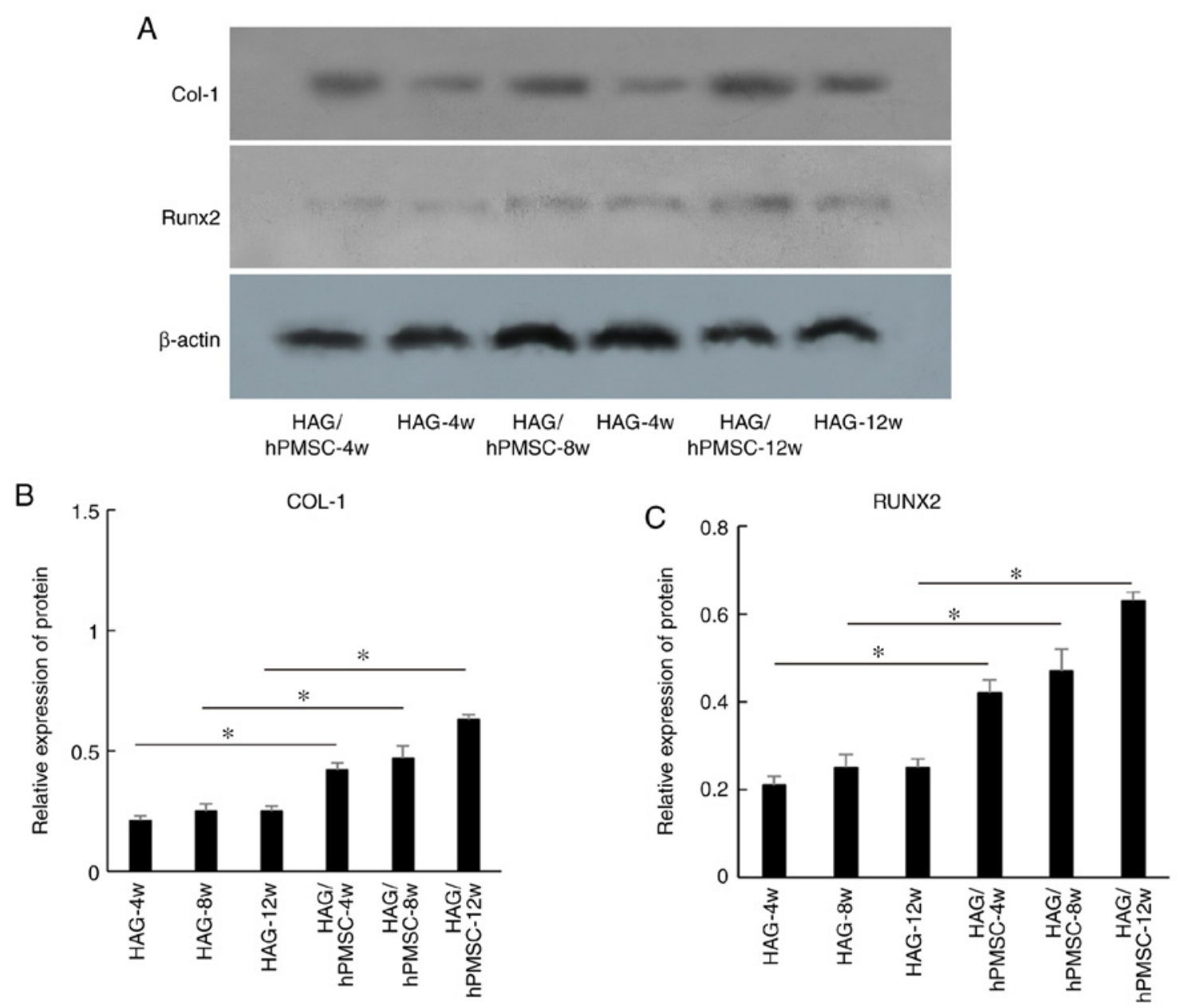

Figure 4. Relative expression of osteogenesis-associated proteins. (A) Relative protein expression of Col-1 and RUNX2 proteins at 4,8 and 12 weeks after implantation of two different scaffolds. (B) Statistical analysis of the relative protein expression of Col-1. (C) Statistical analysis of the relative protein expression of Runx2. "P<0.05. Col-1, collagen-1; Runx2, runt-related transcription factor 2; HAG, hydroxyapatite with a grooved structure; hPMSC, human placenta-derived mesenchymal stem cells; HAG/hPMSCs, HAG scaffold-based delivery of hPMSCs; w, weeks.

following implantation, the areas of bone matrix and mature bone plate in the HAG/hPMSC group were larger than those in the control group, suggesting that hPMSCs may continue to play an important role in the whole process of osteogenesis. At 8 weeks following implantation, pathways associated with cell adhesion, hematopoietic cell lineage, focal adhesion and CAMs played important roles, and osteoclast differentiation was activated. At 12 weeks following implantation, osteogenesis was closely associated with protein digestion and absorption, ECM-receptor interactions, the AMPK signaling pathway and osteoclast differentiation. The AMPK signaling pathway plays a vital role in osteoclast differentiation and aids in improving osteogenesis $(21,29)$. In the present study, differences in the signaling pathways involved in the different stages of osteogenesis were revealed, which are novel findings. 
COL-1 is the main type of collagen secreted by osteoblasts, accounting for $\sim 90 \%$ of the components of the ECM (30). The expression of COL-1 is upregulated following the differentiation of osteoblasts (30). COL-1 is a marker gene for the phenotypic identification and osteogenic differentiation of osteoblasts (31). The results of the present study revealed that the expression of COL-1 increased with time, indicating that bone formation also increased with time. RUNX2, a transcription factor belonging to the Runt family, plays a key role in bone development (32). RUNX2 regulates osteoblast differentiation and osteogenesis during osteogenic differentiation, and the expression level of RUNX2 can also indicate the function and differentiation of osteoblasts (33). The results of the current study are consistent with the qPCR results of a previous study (5). In the present study, the expression level of RUNX2 increased with time, suggesting that osteoblasts continued to differentiate and drive bone formation. However, the present study is limited by the use of only two markers and an increase in the number of marker genes is required for further studies.

In conclusion, the present study preliminarily explored the effect of ectopic osteogenesis of hPMSCs combined with porous HAG scaffolds in vivo. The results of the present study provide the potential for novel applications of this cell type in osteogenesis research.

\section{Acknowledgements}

Not applicable.

\section{Funding}

This work was supported by the Department of Science and Technology of Sichuan Province (grant nos. 2016TD0008 and 2018HH0080) and Chinese National Natural Science Foundation (grant no. 82071168).

\section{Availability of data and materials}

The datasets generated and/or analyzed during the current study are available in the NCBI SRA repository (https://ncbiinsights.ncbi.nlm.nih.gov/tag/sra/) with accession numbers SRR13236657, SRR13236658, SRR13236659, SRR13236660, SRR13236661 and SRR13236662.

\section{Authors' contributions}

YM conceived and designed the study. XR, QW, CL, QZ, JZ and $\mathrm{HX}$ performed the experiments. QW wrote the paper. XR, $\mathrm{KT}$ and YM revised the manuscript and gave final approval of the version to be published. KT reanalyzed the data. YM, QW and KT confirmed the authenticity of all the raw data. All authors have read and approved the final manuscript.

\section{Ethics approval and consent to participate}

All animal experiments were conducted according to the protocols approved by the Animal Care and Use Committee of Sichuan Provincial People's Hospital [Sichuan, P.R. China; approval no. 2019NSF(98)].

\section{Patient consent for publication}

Not applicable.

\section{Competing interests}

The authors declare that they have no competing interests.

\section{References}

1. Cheng X, Lei D, Mao T, Yang S, Chen F and Wu W: Repair of critical bone defects with injectable platelet rich plasma/bone marrow-derived stromal cells composite: Experimental study in rabbits. Ulus Travma Acil Cerrahi Derg 14: 87-95, 2008.

2. Marx RE, Miller RI, Ehler WJ, Hubbard G and Malinin TI: A comparison of particulate allogeneic and particulate autogenous bone grafts into maxillary alveolar clefts in dogs. J Oral Maxillofac Surg 42: 3-9, 1984.

3. Janicki P and Schmidmaier G: What should be the characteristics of the ideal bone graft substitute? Combining scaffolds with growth factors and/or stem cells. Injury 42 (Suppl 2): S77-S81, 2011.

4. Suresh SS, Raniga S, Shanmugam V, George M and Zaki H: Carpal tunnel syndrome due to hydroxyapatite crystal deposition disease. J Hand Microsurg 5: 96-99, 2013.

5. Xiaohua R, Qiang T, Kun T, Huang GT, Li JY, Xu T, Lv X, Wu J, Chen Z, Weng J, et al: Enhancement of osteogenesis using a novel porous hydroxyapatite scaffold in vivo and vitro. Ceram Int 44: 21656-21665, 2018.

6. Wang Z, Gerstein M and Snyder M: RNA-Seq: A revolutionary tool for transcriptomics. Nat Rev Genet 10: 57-63, 2009.

7. Carnes MU, Allingham RR, Ashley-Koch A and Hauser MA: Transcriptome analysis of adult and fetal trabecular meshwork, cornea, and ciliary body tissues by RNA sequencing. Exp Eye Res 167: 91-99, 2018.

8. Cloonan N, Forrest AR, Kolle G, Gardiner BB, Faulkner GJ, Brown MK, Taylor DF, Steptoe AL, Wani S, Bethel G, et al: Stem cell transcriptome profiling via massive-scale mRNA sequencing. Nat Methods 5: 613-619, 2008.

9. Mortazavi A, Williams BA, McCue K, Schaeffer L and Wold B: Mapping and quantifying mammalian transcriptomes by RNA-Seq. Nat Methods 5: 621-628, 2008.

10. Trapnell C, Williams BA, Pertea G, Mortazavi A, Kwan G, van Baren MJ, Salzberg SL, Wold BJ and Pachter L: Transcript assembly and quantification by RNA-Seq reveals unannotated transcripts and isoform switching during cell differentiation. Nat Biotechnol 28: 511-515, 2010.

11. Guttman M, Garber M, Levin JZ, Donaghey J, Robinson J, Adiconis X, Fan L, Koziol MJ, Gnirke A, Nusbaum C, et al: $\mathrm{Ab}$ initio reconstruction of cell type-specific transcriptomes in mouse reveals the conserved multi-exonic structure of lincRNAs. Nat Biotechnol 28: 503-510, 2010.

12. Satija NK, Sharma D, Afrin F, Tripathi RP and Gangenahalli G: High throughput transcriptome profiling of lithium stimulated human mesenchymal stem cells reveals priming towards osteoblastic lineage. PLoS One 8: e55769, 2013.

13. Dominici M, Le Blanc K, Mueller I, Slaper-Cortenbach I, Marini F, Krause D, Deans R, Keating A, Prockop DJ and Horwitz E: Minimal criteria for defining multipotent mesenchymal stromal cells. The international society for cellular therapy position statement. Cytotherapy 8: 315-317, 2006.

14. Squillaro T, Peluso $\mathrm{G}$ and Galderisi U: Clinical trials with mesenchymal stem cells: An Update. Cell Transplant 25: 829-848, 2016.

15. Alessio N, Squillaro T, Özcan S, Di Bernardo G, Venditti M, Melone M, Peluso G and Galderisi U: Stress and stem cells: adult Muse cells tolerate extensive genotoxic stimuli better than mesenchymal stromal cells. Oncotarget 9: 19328-19341, 2018.

16. Fathi E and Farahzadi R: Zinc sulphate mediates the stimulation of cell proliferation of rat adipose tissue-derived mesenchymal stem cells under high intensity of EMF exposure. Biol Trace Elem Res 184: 529-535, 2017.

17. Fathi E, Farahzadi R and Sheikhzadeh N: Immunophenotypic characterization, multi-lineage differentiation and aging of zebrafish heart and liver tissue-derived mesenchymal stem cells as a novel approach in stem cell-based therapy. Tissue Cell 57: 15-21, 2019. 
18. Lim J, Lee J, Yun HS, Park EK and Shin HI: Comparison of bone regeneration rate in flat and long bone defects: Calvarial and tibial bone. Tissue Eng Regene Med 10: 336-340, 2013.

19. Lin YH, Chen CY, Chou LY, Chen CH, Kang L and Wang CZ: Enhancement of bone marrow-derived mesenchymal stem cel osteogenesis and new bone formation in rats by obtusilactone A. Int J Mol Sci 18: 2422, 2017.

20. Livak KJ and Schmittgen TD: Analysis of relative gene expression data using real-time quantitative PCR and the 2(-Delta Delta C(T)) method. Methods 25: 402-408, 2002.

21. Wang YG, Han XG, Yang Y, Qiao H, Dai KR, Fan QM and Tang TT: Functional differences between AMPK alphal and alpha2 subunits in osteogenesis, osteoblast-associated induction of osteoclastogenesis, and adipogenesis. Sci Rep 6: 32771, 2016.

22. Shu C, Zhao M, Anderson JP, Garg G, Singh KB, Zheng W, Wang C, Yang $M$ and Zhou E: Transcriptome analysis reveals molecular mechanisms of sclerotial development in the rice sheath blight pathogen Rhizoctonia solani AG1-IA. Funct Integr Genomics 19: 743-758, 2015

23. Liu Z, Yin X, Ye Q, He W and Zou S: Periodontal regeneration with stem cells-seeded collagen-hydroxyapatite scaffold. J Biomater Appl 31: 121, 2016.

24. Wang G, Yang H, Li M, Lu S, Chen X and Cai X: The use of silk fibroin/hydroxyapatite composite co-cultured with rabbit bone-marrow stromal cells in the healing of a segmental bone defect. Bone Joint Surg Br 92: 320-325, 2010.

25. Diao Y, Ma Q, Cui F and Zhong Y: Human umbilical cord mesenchymal stem cells: Osteogenesis in vivo as seed cells for bone tissue engineering. J Biomed Mater Res Part A 91A: $123-131,2010$

26. Liu Z, Yin X, Ye Q, He W, Ge M, Zhou X, Hu J and Zou S: Periodontal regeneration with stem cells-seeded collagen-hydroxyapatite scaffold. J Biomater Appl 31: 121-131, 2016.
27. Zhou L, Chen J, Li Z, Li X, Hu X, Huang Y, Zhao X, Liang C, Wang Y, Sun L, et al: Integrated profiling of microRNAs and mRNAs: microRNAs located on Xq27.3 associate with clear cell renal cell carcinoma. PLoS One 5: e15224, 2010.

28. Hung SC, Pochampally RR, Chen SC, Hsu SC and Prockop DJ Angiogenic effects of human multipotent stromal cell conditioned medium activate the PI3K-Akt pathway in hypoxic endothelial cells to inhibit apoptosis, increase survival, and stimulate angiogenesis. Stem Cells 25: 2363-2370, 2007.

29. Wang YG, Qu XH, Yang Y, Han XG, Wang L, Qiao H, Fan QM, Tang TT and Dai KR: AMPK promotes osteogenesis and inhibits adipogenesis through AMPK-Gfi1-OPN axis. Cell Signal 28: 1270-1282, 2016

30. Gupta V, Shah MA, Shah SK and Shah JM: Osteoanabolics. Indian J Endocrinol Metab 6: 349-357, 2012.

31. Zheng J, Mao X, Ling J, Chen C and Zhang W: Role of magnesium transporter subtype 1 (MagT1) in the osteogenic differentiation of rat bone marrow stem cells. Biol Trace Elem Res 171: 131-137, 2016.

32. Cui CB, Cooper LF, Yang X, Karsenty G and Aukhil I: Transcriptional coactivation of bone-specific transcription factor Cbfa1 by TAZ. Mol Cell Biol 23: 1004-1013, 2003.

33. Kwun IS, Cho YE, Lomeda RA, Shin HI, Choi JY, Kang YH and Beattie JH: Zinc deficiency suppresses matrix mineralization and retards osteogenesis transiently with catch-up possibly through Runx 2 modulation. Bone 46: 732-741, 2010.

(i) $\odot$ This work is licensed under a Creative Commons Attribution-NonCommercial-NoDerivatives 4.0 International (CC BY-NC-ND 4.0) License. 